\title{
Understanding Iron Deficiency and Exercise: Looking Beyond Ferritin
}

\author{
Richard J. Burden ${ }^{\mathrm{a}, \mathrm{b}} \quad$ Charles R. Pedlar ${ }^{\mathrm{a}}$ \\ ${ }^{a}$ St Mary's University, Twickenham, UK; ${ }^{\text {b }}$ English Institute of Sport, Manchester, UK
}

Iron plays a crucial role in multiple physiological functions and is essential for human health and physical performance, particularly for athletes or military personnel whose iron requirements may be higher due to factors like increased erythropoietic drive, footstrike hemolysis, menses, gastrointestinal bleeding, exercise-induced inflammation, the use of anti-inflammatory drugs, and environmental stress. Investigations into the effects of strenuous physical activity on iron status have predominantly concentrated on females, but in this issue of Acta Haematologica, Epstein et al. [1] describe the development of iron deficiency (ID) and iron deficiency anemia (IDA) in male soldiers. The prevalence of ID and IDA in recruited males was unexpectedly high. It increased significantly following the first 6 months of training, and was attributed to the continuous physical training. Whilst there is an awareness of ID in females, this report by Epstein et al. [1] shows that males undertaking arduous military training are also vulnerable to ID. Attention should be paid to the hematological monitoring and nutritional status of male soldiers and athletes to ensure that the detrimental effects of ID are recognized and treated appropriately.

\section{KARGER}

(C) 2018 S. Karger AG, Basel

E-Mail karger@karger.com

www.karger.com/aha
ID and IDA prevalence data are influenced by the diagnostic criteria applied. For example, Epstein et al. [1] used the cut-off values $<14 \mathrm{~g} / \mathrm{dL}$ for hemoglobin $(\mathrm{Hb})$ concentration and $<30 \mu \mathrm{g} / \mathrm{L}$ for serum ferritin (sFer) to define ID and IDA. They acknowledge that this definition is in contrast to that used in much of the literature.

Correctly diagnosing and treating ID in athletes is a challenge, due to: (1) the absence of a clear definition; (2) the absence of a clear treatment and monitoring protocol, particularly if the ID is not accompanied by anemia; (3) training-induced plasma volume expansion. Furthermore, the level of sFer is the most commonly used indicator of iron status. However, this value varies throughout the literature where a range of $12-40 \mu \mathrm{g} / \mathrm{L}$ can be considered as ID [2] whereas in clinical investigations of inflammatory diseases, $<100 \mu \mathrm{g} / \mathrm{L}$ might be viewed as ID. The acute-phase response of sFer adds further complications, as exercise or illness can cause variations, potentially masking the true value.

Epstein et al. [1] and Kell and Pretorius [3] question the use of sFer for monitoring the iron status of individuals who undergo physical training. Traditionally, sFer is used as an indicator of whole-body iron status based on 
tissue ferritin correlating strongly with iron stores. sFer, however, actually lacks most of the iron it contained in its intracellular form. A more contemporary understanding of sFer is as a marker of inflammation and cell death [3].

Alternative markers are needed. Epstein et al. [1] suggest that transferrin saturation (TSAT) and transferrin receptor level are useful additions to sFer. TSAT can be influenced by inflammation, but the soluble transferrin receptor is not an acute-phase protein. Total $\mathrm{Hb}$ mass measured by carbon monoxide rebreathing has also been used to assess the effectiveness of iron treatments [4].

Complete blood cell count indices, assessed alongside $\mathrm{Hb}$, sFer, and TSAT, supply information about the impact that ID and IDA may be having on reticulocyte and erythrocyte populations. These measurements remain stable in healthy individuals but marked alterations occur in chronic disease states [5]. However, unlike in IDA, complete blood cell count indices remain unchanged in ID without anemia, thus leaving the understanding of this condition largely incomplete.

Hepcidin, a peptide hormone that regulates iron absorption, shows promise as a key marker of iron status. The influence of strenuous physical activity on hepcidin should be a target of future research. The regulation of hepcidin is mediated by multiple stimuli, including iron status, inflammation, and hypoxia, which are relevant in the case of soldiers and athletes. Longitudinal measure- ments of acute and chronic exercise exposure, together with these biomarker variables, would further our understanding of how physical training may affect iron absorption.

Currently, ID can only be confirmed by a positive response to treatment, in the form of an increase in total $\mathrm{Hb}$ mass, but this retrospective approach still does not allow practitioners to be proactive in the identification and treatment of conditions related to ID. Given that traditional methods of assessing ID, particularly ID without anemia, do not provide a definitive insight into the condition, it is perhaps time to seek an alternative. Recently, a model has been developed which characterizes the birth rate, maturation, and clearance dynamics of red blood cell populations [6]. It uses raw data from automated hemoanalyzers that report single reticulocyte and erythrocyte volume and $\mathrm{Hb}$ measurements. This study by Higgins and Mahadevan [6] identified patients with anemia and distinguished thalassemia-trait anemia from IDA. The model also identifies preanemic states several weeks before anemia has become clinically detectable. Their research was based on clinical populations, but the findings could have important implications for the athletics setting. Early identification of iron-deficient states would be significant for the support of the health, well-being, and performance of military personnel and athletes. It is time for the research into ID to look beyond ferritin.

\section{References}

Epstein D, Borohovitz A, Merdler I, Furman M, Attali E, Sorkin A, Stainfeld Y, Isenberg Y, Mashiach T, Shapira S, Weisshof R, Dann EJ: Prevalence of iron deficiency and iron deficiency anemia in strenuously training male army recruits. Acta Haematol 2018, DOI: 10.1159/000485736.

-2 Burden RJ, Morton K, Richards T, Whyte GP, Pedlar CR: Is iron treatment beneficial in iron-deficient but non-anaemic (IDNA) endurance athletes? A meta-analysis. Br J Sports Med 2014;49:1-10.
Kell DB, Pretorius E: Serum ferritin is an important inflammatory disease marker, as it is mainly a leakage product from damaged cells. Metallomics 2014;6:748-773.

4 Wachsmuth NB, Aigner T, Völzke C, Zapf J, Schmidt WF: Monitoring recovery from iron deficiency using total hemoglobin mass. Med Sci Sports Exerc 2015;47:419-427.
Brugnara C, Mohandas N: Red cell indices in classification and treatment of anemias: from M.M. Wintrobes's original 1934 classification to the third millennium. Curr Opin Hematol 2013;20:222-230.

6 Higgins JM, Mahadevan L: Physiological and pathological population dynamics of circulating human red blood cells. Proc Natl Acad Sci USA 2010;107:20587-20592. 